\title{
中間漸移層がる場合の弾性波の反射及ひ透過
}

\author{
東京头学理学部地球物理学教室 佐藤良輔 \\ (昭和 32 年 10 月 8 日受理)
}

\section{Reflection and Transmission of Elastic Waves at a Discontinuity with an Intervenient Transient Layer.}

\author{
Ryôsuke SATô \\ Geophysical Institute, Faculty of Science, Tokyo University.
}

(Received Oct. 8, 1957)

\begin{abstract}
Reflection and refraction of elastic waves at a discontinuous boundary in transmitting medium have been studied by many authors since 18991). Most of them have assumed that density or elastic constant of the medium vary discontinuously (mathematically) at the boundary. While there can be little doubt that very sharp discontinuities exist in the earth, it will be better to consider that physical properties of materials in the immediate vicinity of the "discontinuity" vary continuously in actual cases. In such cases, the most pertinent transfer of energy among the waves concerned takes place near the boundary, say, within a few wave-lengths from it, and no theory can be adequate without referring to the sharpness of discontinuity as compared with the wave-length.

K. Sezawa and K. Kanai ${ }^{2}$, in 1935 , and A. Wolf ${ }^{3}$, in 1937 , are the only two who investigated the effect of sharpness of discontinuity on reflection and refraction phenomena.

Their studies, however, were confined only to vertical incidence of waves, (although, in the latter, some qualitative statements were made about the general case), with simplifying assumptions that densities are the same everywhere and rigidity or velocity in the intervenient layer varies linearly with depth.

In this paper, the present writer has investigated reflection and transmission of elastic waves at "discontinuity" for the incidence of SH-waves with various glancing angles, supposing that there is an invervenient layer of thickness $H$ between two different media. In the intervenient layer, both density and rigidity vary continuously but steeply from the corresponding values of the upper medium to those of the lower one. He has found that the reflected wave is considerably affected by the existence of the intervenient layer if the wave-length of incident wave is very small compared with the thickness of the layer, but the transmitted wave is not affected very much.
\end{abstract}

\section{§ 1. 序 論}

弾性媒質内部の不連続面に和ける弾性波の反射, 屈折の問題は, Knott ${ }^{1)}$ (1899) 以来その殆 んぞ全てが媒質の性質が完全に不連続に（数学的に）变化する場合を取扱つている. 実際相当 に著しい变化のある面のあるのは地震波等の観測から衆知のことである.乙かし実際の地球内 部では, 現在理論で取扱つているような, 完全に不連続に変るような面よりる, 寧ろ急激では あるが連続的に性質が变るような構造の方が考兄易い。この不連続の程度に較べて, 弾性波の 波長が同程度か短いような場合は, 当然相当大きな影響があらわれることが予想されるわけで 
ある。

このような問題を取扱つたのは，僅かに妹沢・金井2)と，A. Wolf ${ }^{32}$ だけであるが，両論文 とも, 波が不連続面に直角に入射する場合に限られ（後者は, 斜めの入射について若干の定性 的な議論をしてはいるが), 然も中間の漸移層で密度は一定, 剛性率或いは速度だけが直線的に 変化する場合についてだけであつた，著者は 2 つ異つた媒質 I , III の中間に層 II を考光, その中では密度, 剛性率が共に連続的ではあるが急激に I 層の值から III 層の值に移るもの とした。そのような、“不連続面”に色々な角度で SH波が入射するとき，反射波拈よび透過波 の振幅が，入射波の波長の大小によつてどの程度变るかを調べたのである.

\section{§ 2. 不均質媒質における解}

変位の $y$ 成分を $v$, 密度, 剛性率を $\rho, \mu$ とし, この $\rho, \mu$ の変化が $z$ 方向にだけとすると, $\mathrm{SH}$ 波についての運動方程式は,

$$
\rho \frac{\partial^{2} v}{\partial t^{2}}=\mu \frac{\partial^{2} v}{\partial x^{2}}+\mu \frac{\partial^{2} v}{\partial z^{2}}+\frac{d \mu}{d z} \frac{\partial v}{\partial z}
$$

で与光られる。今

$$
v=\frac{1}{\sqrt{\mu}} Z(z) e^{-i \omega t+i \xi x}
$$

と置くと，

$$
\frac{d^{2} Z}{d z^{2}}-\left[\xi^{2}-\frac{\rho \omega^{2}}{\mu}+\frac{1}{2 \mu} \frac{d^{2} \mu}{d z^{2}}-\frac{1}{4}\left\{\frac{1}{\mu} \frac{d \mu}{d z}\right\}^{2}\right] Z=0
$$

となりここでヌ

$$
\sqrt{\mu / \rho}=V(z), \omega / V=k(z), k_{1} z=\zeta, \xi / k_{1}=\gamma, k / k_{1}=\kappa(z),
$$

とすると，(3) は結局

$$
\frac{d^{2} Z}{d \zeta^{2}}-\left[\gamma^{2}-\kappa^{2}+\frac{\mu^{\prime \prime}}{2 \mu}-\frac{1}{4}\left(\frac{\mu^{\prime}}{\mu}\right)^{2}\right] Z=0
$$

そ変形される。 ただし $\mu^{\prime}=d \mu / d \zeta=k_{1}^{-1}(d \mu / d z), \quad \mu^{\prime \prime}=d^{2} \mu / d \zeta^{2}=k_{1}^{-2}\left(d^{2} \mu / d z^{2}\right)$ である。

（5）の解は次のように書くことができる.

$$
Z=C Z^{(1)}(\zeta)+D Z^{(2)}(\zeta)=C \sum_{0}^{\infty} Z_{n}^{(1)}(\zeta)+D \sum_{0}^{\infty} Z_{n}^{(2)}(\zeta),
$$

ここで

$$
\left.\begin{array}{l}
Z_{0}^{(1)}=1, Z_{0}^{(2)}=\zeta=k_{1} z \\
Z_{n}^{(1),(2)}(\zeta)=-\int_{0}^{\zeta}(\lambda-\zeta)\left[\gamma^{2}-\kappa^{2}(\lambda)+\frac{\mu^{\prime \prime}(\lambda)}{2 \mu(\lambda)}-\frac{1}{4}\left\{\frac{\mu^{\prime}(\lambda)}{\mu(\lambda)}\right\}^{2}\right] Z_{n-1}^{(1),(2)}(\lambda) d \lambda \quad n \geqslant 1
\end{array}\right\}
$$




$$
\mu^{\prime}(\lambda)=\frac{1}{k_{1}}\left[\frac{d \mu}{d z}\right]_{z=\lambda / k_{1}}, \quad \mu^{\prime \prime}=\frac{1}{k_{1}^{2}}\left[\frac{d^{2} \mu}{d z^{2}}\right]_{z=\lambda / k_{1}}
$$

これが (5) の解であり, また (5) の[ ] 内が考它る領域で有限である時は, $\sum_{0}^{\infty} Z_{n}^{(1),(2)}$ が一様収斂する ことは次のようにして証明される $\left[Z^{(1)}\right.$ についても， $Z^{(2)}$ につても同じであるから，今は $Z^{(1)}$ といて だけ証明し，また簢単の為肩の (1) は省く].

今 (5) を

$$
d^{2} Z / d \zeta^{2}-F(\zeta) \cdot Z=0
$$

とすると;（7）から

$$
d^{2} Z_{n} / d \zeta^{2}=F(\zeta) \cdot Z_{n-1}(\zeta)
$$

従つて,

$$
\frac{d^{2} Z}{d \zeta^{2}}=\frac{d^{2} Z_{0}}{d \zeta^{2}}+\frac{d^{2}}{d \zeta^{2}} \sum_{1}^{\infty} Z_{n}=F(\zeta) \sum_{1}^{\infty} Z_{n-1}(\zeta)=F(\zeta) \cdot Z(\zeta)
$$

となり（8）は（5）を満足する，また収斂性は，今考光る領域行ける $\left|Z_{0}\right|$ 特よび $|F(\zeta)|$ の最大值を それぞれ $M, N$ とし，

$$
\left|Z_{n}(\zeta)\right| \leqslant M N^{n}|\zeta|^{2 n} / n !
$$

と仮定すると，これは $n=0$ の時確かに成立する. そこで $n=1,2,3, \cdots, m-1$ 飞対して成立したとす ると，

$$
\begin{aligned}
\left|Z_{m}(\zeta)\right| & =\left|\int_{0}^{\zeta}(\lambda-\zeta) F^{\prime}(\lambda) Z_{m-1}(\lambda) d \lambda\right| \leqslant \frac{1}{(m-1) !} \int_{0}^{\zeta}|\lambda-\zeta| M N^{m}|\lambda|^{2 m-2} d \lambda \\
& \leqslant \frac{M N^{m}}{(m-1) !}|\zeta| \int_{0}^{\zeta}|\lambda|^{m-2} d \lambda
\end{aligned}
$$

$m \geqslant 1$ の時は, $2 m-1 \geqslant m$ であるから, 結局

即ち考觉る領域で $Z=\sum_{0}^{\infty} Z_{n}(\zeta)$ は一様収斂する.

$$
\left|Z_{m}(\zeta)\right| \leqslant \frac{M N^{m}|\zeta|^{2 m}}{m !}
$$

\section{§3. 反射波および透過波の振幅}

媒質 I , III は，密度，剛性率，速度がそれぞれ $\rho_{1}, \mu_{1}, V_{1}$ 特よび $\rho_{2}, \mu_{2}, V_{2}$ であるような均 質完全弾性体とし $\left(V_{1}<V_{2}\right.$ として和く)，亦 た I と III にはさまれた厚さ $H$ の媒質 II は, 上のような性質が I から III へ連続的に変つて 行くような不均質層とする，そして波がIから II へ入射角 $\theta_{1}$ で入射し， II から III へ屈折角 $\theta_{2}$ で透過する場合，扣よびそれと逆に， III か ら II 几入射角 $\theta_{2}$ で入射し，川から I へ屈折 角 $\theta_{1}$ で透過する場合を考光る。

$\mathrm{I}$ と II の境界面を $x y$ 面, $z$ 軸を I から III へ 向けてとる. 3 つの媒質に拈ける解を次のよう

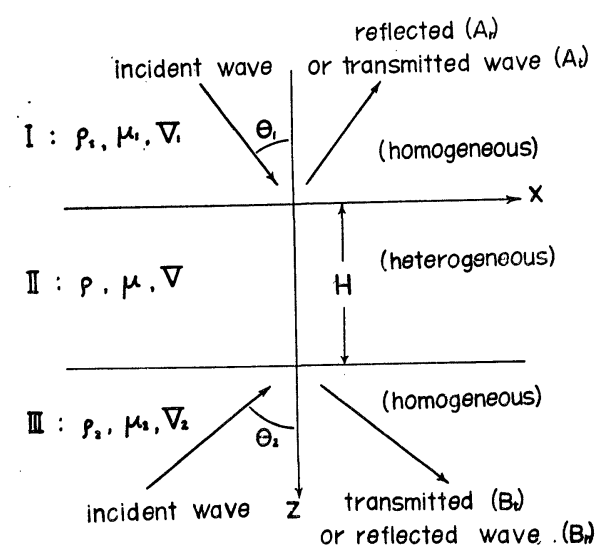

Fig. 1 
に特く。

媒質 I で, $\quad v_{1}=\alpha e^{i k_{1}\left(x \sin \theta_{1}+z \cos \theta_{1}\right)}+A e^{i k_{1}\left(x \sin \theta_{1}-z \cos \theta_{1}\right)}$,

媒質 II で， $\quad v_{2}=\frac{1}{\sqrt{\mu}}\left[C Z^{(1)}(\zeta)+D Z^{(2)}(\zeta)\right] e^{i \xi x}$ ，

媒質 III で, $\quad v_{3}=\beta e^{i k_{2}\left(x \sin \theta_{2}-z \cos \theta_{2}\right)}+B e^{i k_{2}\left(x \sin \theta_{2}+z \cos \theta_{2}\right)}$.

ただし，

$$
k_{1}{ }^{2}=\rho_{1} \omega^{2} / \mu_{1}=\omega^{2} / V_{1}{ }^{2}, \quad k_{2}{ }^{2}=\rho_{2} \omega^{2} / \mu_{2}=\omega^{2} / V_{2}{ }^{2}
$$

で, time factor は省略, また (10)の $Z^{(1)}(\zeta), Z^{(2)}(\zeta)$ は (7) で与えられるるので女り, $\zeta=k_{1} z$ である.

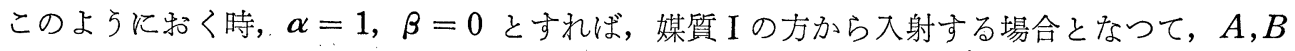
がそれぞれ反射波拈よび透過波の振幅，また $\alpha=0, \beta=1$ とすれば，逆に媒質 III からの入 射で, $B$ が反射波， $A$ が透過波の振幅となるわけである.

境界条件は,

$$
\left.\begin{array}{lll}
z=0(\zeta=0) \text { で, } \quad \partial v_{1} / \partial z=\partial v_{2} / \partial z, & v_{1}=v_{2}, \\
z=H\left(\zeta=k_{1} H\right) \text { で, } \quad \partial v_{2} / \partial z=\partial v_{3} / \partial z, & v_{2}=v_{3} .
\end{array}\right\}
$$

従つて, $\xi=k_{1} \sin \theta_{1}=k_{2} \sin \theta_{2}$ なる事を考慮すると， 結局 $A, B, C, D$ を決める式として， $i k_{1} \cos \theta_{1} \cdot A$

$$
+\frac{k_{1}}{\sqrt{\mu_{1}}} E_{0}^{(1)} \cdot C+\frac{k_{1}}{\sqrt{\mu_{1}}} E_{0}^{(2)} \cdot D=i k_{1} \cos \theta_{1} \cdot \alpha
$$

$-A$

$$
+\frac{1}{\sqrt{\mu_{1}}} Z_{0}^{(1)} \cdot C+\frac{1}{\sqrt{\mu_{1}}} Z_{0}^{(2)} \cdot D=\alpha
$$

$-i k_{2} \cos \theta_{2} e^{i k_{2} H \cos \theta_{2}} \cdot B+\frac{k_{1}}{\sqrt{\mu_{2}}} E_{H}^{(1)} \cdot C+\frac{k_{1}}{\sqrt{\mu_{2}}} E_{H}^{(2)} \cdot D=-i k_{2} \cos \theta_{2} e^{-i k_{2} H \cos \theta_{2}} \cdot \beta$

$$
-e^{i k_{2} H \cos \theta_{2}} \cdot B+\frac{1}{\sqrt{\mu_{2}}} Z_{H}^{(1)} \cdot C+\frac{1}{\sqrt{\mu_{2}}} Z_{H}^{(2)} \cdot D=e^{-i k_{2} H \cos \theta_{2}} \cdot \beta .
$$

ただし，

$$
E^{(1),(2)}=\frac{d Z^{(1),(2)}}{d \zeta}-\frac{1}{2 k_{1}}\left[\frac{1}{\mu} \frac{d \mu}{d z}\right] Z^{(1),(2)}
$$

で, suffix $0, H$ はそれぞれ $z=0, H$ (即ち $\zeta=0, k_{1} H$ ) に和ける值を示するので女る.

（14）から $A, B$ を求めると, 結局,

$$
\left.\begin{array}{l}
A \Delta=-\alpha[(X-Y)-i(U+W)]+2 i \beta e^{-i k_{2} H \cos \theta_{2}} \cdot S, \\
B \Delta=2 i \alpha e^{-i k_{2} H \cos \theta_{2}} \cdot R-\beta e^{-2 i k_{2} H \cos \theta_{2}}[(X-Y)+i(U+W)] .
\end{array}\right\}
$$

ここで, 


$$
\begin{aligned}
\Delta & =(X+Y)+i(U-W), \\
X & =\frac{k_{1}}{k_{2}}\left[E_{0}^{(1)} E_{H}^{(2)}-E_{0}^{(2)} E_{H}^{(1)}\right], \\
Y & =\left[Z_{0}^{(1)} Z_{H}^{(2)}-Z_{0}^{(2)} Z_{H}^{(1)}\right] \cos \theta_{1} \cdot \cos \theta_{2}, \\
U & =\frac{k_{1}}{k_{2}}\left[Z_{0}^{(1)} E_{H}^{(2)}-Z_{0}^{(2)} E_{H}^{(1)}\right] \cos \theta_{1}, \\
W & =\left[E_{0}^{(1)} Z_{H}^{(2)}-E_{0}^{(2)} Z_{H}^{(1)}\right] \cos \theta_{2}, \\
R & =\sqrt{\frac{\mu_{1}}{\mu_{2}}} \frac{k_{1}}{k_{2}}\left[Z_{H}^{(1)} E_{H}^{(2)}-Z_{H}^{(2)} E_{H}^{(1)}\right] \cos \theta_{1}, \\
S & =\sqrt{\frac{\mu_{2}}{\mu_{1}}}\left[Z_{0}^{(1)} E_{0}^{(2)}-Z_{0}^{(2)} E_{0}^{(1)}\right] \cos \theta_{2} .
\end{aligned}
$$

ただし， $Z_{0}^{(1)}=1, Z_{0}^{(2)}=0$ ，従つて $E_{0}^{(1)}=-\frac{1}{2 k_{1}}\left[\frac{1}{\mu} \frac{d \mu}{d z}\right]_{0}, E_{0}^{(2)}=1$ である.

かくして，それぞれの場合に対する反射波扣よび透過波の振幅は次のようになる.

1）媒質 I の側から入射する場合（入射角 $\theta_{1}$ ).

a) $\theta_{1}<\sin ^{-1}\left(V_{1} / V_{2}\right)$ のとき.

$$
\left.\begin{array}{ll}
\left|A_{r}\right|=\sqrt{\frac{(X-Y)^{2}+(U+W)^{2}}{(X+Y)^{2}+(U-W)^{2}}} & \text { (反射波)， } \\
\left|B_{t}\right|=\frac{2 R}{\sqrt{(X+Y)^{2}+(U-W)^{2}}} & \text { (透過波). }
\end{array}\right\}
$$

b) $\theta_{1}>\sin ^{-1}\left(V_{1} / V_{2}\right)$ のとき.

このときは, $\cos \theta_{2}$ は純虚数, 即ち $\cos \theta_{2}=i \Theta$ となるから, $Y=i Y^{\prime}, W=i W^{\prime}$ と抒 $<\varepsilon$,

$$
\left.\begin{array}{l}
\left|A_{r}\right|=\sqrt{\frac{\left(X+W^{\prime}\right)^{2}+\left(Y^{\prime}+U\right)^{2}}{\left(X+W^{\prime}\right)^{2}+\left(Y^{\prime}+U\right)^{2}}=1} \quad \text { (反射波)，} \\
\left|B_{t}\right| e^{-k_{2} \Theta H}=\frac{2 R}{\sqrt{\left(X+W^{\prime}\right)^{2}+\left(Y^{\prime}+\bar{U}^{2}\right.}} \text { (透過波). }
\end{array}\right\}
$$

2) 媒質 III の側から入射する場合（入射角 $\theta_{2}$ ).

$$
\left.\begin{array}{ll}
\left|A_{t}\right|=\frac{2 S}{\sqrt{(X+Y)^{2}+(U-W)^{2}}} & \text { (透過波)， } \\
\left|B_{r}\right|=\sqrt{\frac{(X-Y)^{2}+(U+W)^{2}}{(X+Y)^{2}+(U-W)^{2}}} & \text { (反射波). }
\end{array}\right\}
$$

§ 4. $\mu=\mu_{1} e^{2 a z}, \quad V=V_{1} e^{b z}$ のとき.

このようなとき, 密度は $\rho=\rho_{1} e^{2 c z}(c=a-b)$ で, また媒質 III に和ける剛性率, 速度は 
$\mu_{2}=\mu_{1} e^{2 a H}, \quad V_{2}=V_{1} e^{b H}$ となる.

方程式 (5) は,

ただし，

$$
d^{2} Z / d \zeta^{2}-\left[p^{2}-e^{-2 q \zeta}\right] Z=0 .
$$

$$
\left.\begin{array}{l}
p^{2}=\gamma^{2}+\left(a^{2} / k_{1}^{2}\right)=\sin ^{2} \theta_{1}+\left(a^{2} / k_{1}^{2}\right), \\
q=b / k_{1} .
\end{array}\right\}
$$

（22）の解は， $C_{\nu}$ をレ次の円壔函数とすると，

$$
Z=C_{p / q}\left[e^{-q \zeta} / q\right]
$$

の形で与えられるが，ここでは ミ2 で解いたように，

$$
\left.\begin{array}{l}
Z_{0}^{(1)}=1, Z_{0}^{(2)}=\zeta, \\
Z_{n}^{(1),(2)}(\zeta)=-\int_{0}^{\zeta}(\lambda-\zeta)\left[p^{2}-e^{-2 q \lambda}\right] Z_{n-1}^{(1),(2)}(\lambda) d \lambda
\end{array}\right\}
$$

と打くと，

$$
\begin{aligned}
& Z_{1}^{(1)}=\frac{p^{2} \zeta^{2}}{2 !}-\frac{\zeta}{2 q}+\frac{1}{(2 q)^{2}}-\frac{1}{(2 q)^{2}} e^{-2 q \zeta}=\frac{\left(p^{2}-1\right) \zeta^{2}}{2 !}+\zeta^{2} \sum_{1}^{\infty} \frac{(-)^{n+1}(2 q \zeta)^{n}}{(n+2) !}, \\
& Z_{1}^{(2)}=\frac{p^{2} \zeta^{2}}{3 !}-\frac{\zeta}{(2 q)^{2}}+\frac{2}{(2 q)^{3}}-\frac{\zeta}{(2 q)^{2}} e^{-2 q \zeta}-\frac{2}{(2 q)^{3}} e^{-2 q \zeta}=\frac{\left(p^{2}-1\right) \zeta^{3}}{3 !}+\zeta^{3} \sum_{1}^{\infty} \frac{(-)^{n+1}(2 q \zeta)^{n}}{n !(n+2)(n+3)}, \\
& Z_{2}^{(1)}=\frac{p^{4} \zeta^{4}}{4 !}-\frac{p^{2} \zeta^{3}}{3 !(2 q)}+\frac{p^{2} \zeta^{2}}{2 !(2 q)^{2}}-\left(2 p^{2}-\frac{1}{2}\right) \frac{\zeta}{(2 q)^{3}}+\left(4 p^{2}-5\right) \frac{1}{(2 q)^{4}} \\
& -\left[\frac{p^{2} \zeta^{2}}{2(2 q)^{2}}+\left(2 p^{2}-1\right) \frac{\zeta}{(2 q)^{3}}+\left(4 p^{2}-1\right) \frac{1}{(2 q)^{4}}\right] e^{-2 q \zeta}+\frac{e^{-4 q \zeta}}{4(2 q)^{4}} \\
& =\frac{\left(p^{2}-1\right)^{2} \zeta^{4}}{4 !}+\frac{\left(p^{2}-1\right) \zeta^{4}}{2 !} \sum_{1}^{\infty} \frac{(-)^{n+1}(2 q \zeta)^{n}}{n !(n+3)(n+4)}+\left(p^{2}-1\right) \zeta^{4} \sum_{1}^{\infty} \frac{(-)^{n+1}(2 q \zeta)^{n}}{(n+4) !} \\
& +\zeta^{4} \sum_{1}^{\infty} \frac{(-)^{n+1}}{(n+2) !} \sum_{1}^{\infty} \frac{(-)^{s+1}(2 q \zeta)^{n+s}}{s !(n+s+3)(n+s+4)} \text {, } \\
& Z_{2}^{(2)}=\frac{p^{4} \zeta^{5}}{5 !}-\frac{p^{2} \zeta^{3}}{3 !(2 q)^{2}}+\frac{p^{2} \zeta^{2}}{(2 q)^{3}}-\left(4 p^{2}-\frac{1}{4}\right) \frac{\zeta}{(2 q)^{4}}+\left(8 p^{2}-\frac{3}{4}\right) \frac{1}{(2 q)^{5}} \\
& -\left[\frac{p^{2} \zeta^{3}}{6(2 q)^{2}}+\frac{p^{2} \zeta^{2}}{(2 q)^{3}}+\left(4 p^{2}-1\right) \frac{\zeta}{(2 q)^{4}}+\frac{8 p^{2}}{(2 q)^{5}}\right] e^{-2 q \zeta}+\left[\frac{\zeta}{4(2 q)^{4}}+\frac{3}{4(2 q)^{5}}\right] e^{-4 q \zeta} \\
& =\frac{\left(p^{2}-1\right)^{2} \zeta^{5}}{5 !}+\frac{\left(p^{2}-1\right) \zeta^{5}}{3 !} \sum_{1}^{\infty} \frac{(-)^{n+1}(2 q \zeta)^{n}}{n !(n+4)(n+5)}+\left(p^{2}-1\right) \zeta^{5} \sum_{1}^{\infty} \frac{(-)^{n+1}(n+1)(2 q \zeta)^{n}}{(n+5) !} \\
& +\zeta^{5} \sum_{1}^{\infty} \frac{(-)^{n+1}}{n !(n+2)(n+3)} \sum_{1}^{\infty} \frac{(-)^{s+1}(2 q \zeta)^{n+s}}{s !(n+s+4)(n+s+5)}, \\
& Z_{3}^{(1)}=\frac{\left(p^{2}-1\right)^{3} \zeta^{6}}{6 !}+\cdots \cdots, \\
& Z_{3}^{(2)}=\frac{\left(p^{2}-1\right)^{3} \zeta^{7}}{7 !}+\cdots \cdots,
\end{aligned}
$$


のように解が得られる.

さて, 今の場合 $2 a H=\log \left(\mu_{2} / \mu_{1}\right), 2 b H=2 \log \left(V_{2} / V_{1}\right)$ であるから，これらを一定の低 $k_{1} H \rightarrow 0$ とすると, 上式から

$$
\begin{aligned}
& Z_{H}^{(1)} \rightarrow 1+(a H)^{2} / 2 !+(a H)^{4} / 4 !+\cdots \cdots=\cosh (a H), \\
& \left.\left.Z_{H}^{(2)} /\left(k_{1} H\right) \rightarrow 1+(a H)^{2} / 3 !+(a H)^{4} / 5 !+\cdots \cdots=\sinh (a H) /\right) a H\right), \\
& \left(k_{1} H\right) d Z_{H}^{(1)} / d \zeta \rightarrow(a H)^{2}+(a H)^{4} / 3 !+\cdots \cdots=(a H) \sinh (a H), \\
& d Z_{H}^{(2)} / d \zeta \rightarrow 1+(a H)^{2} / 2 !+(a H)^{4} / 4 !+\cdots \cdots=\cosh (a H) .
\end{aligned}
$$

従つて,

$$
\left(k_{1} H\right) E_{H}^{(1)} \rightarrow-(a H) e^{-a H}, E_{H}^{(2)} \rightarrow e^{-a H},
$$

となるから，結局（18）の各式は

$$
\left.\begin{array}{l}
X \rightarrow 0, \quad Y \rightarrow 0, \quad U \rightarrow \frac{k_{1}}{k_{2}} e^{-a \cdot H} \cos \theta_{1}=\frac{k_{1}}{k_{2}} \sqrt{\mu_{1}} \cos \theta_{1} \\
W \rightarrow-e^{a H} \cos \theta_{2}=-\sqrt{\mu_{2}} \cos \theta_{2}, \quad R \rightarrow \frac{k_{1}}{k_{2}} \sqrt{\frac{\mu_{1}}{\mu_{2}}} \cos \theta_{1}, S \rightarrow \sqrt{\frac{\mu_{2}}{\mu_{1}}} \cos \theta_{2},
\end{array}\right\}
$$

となり, これから $A_{r}, t, B_{r, t}$ を出すと，

$$
\left.\begin{array}{l}
A_{r}=\frac{\mu_{1} k_{1} \cos \theta_{1}-\mu_{2} k_{2} \cos \theta_{2}}{\mu_{1} k_{1} \cos \theta_{1}+\mu_{2} k_{2} \cos \theta_{2}}=-B_{r} \\
\frac{B_{t}}{\mu_{1} k_{1} \cos \theta_{1}}=\frac{2}{\mu_{1} k_{1} \cos \theta_{1}+\mu_{2} k_{2} \cos \theta_{2}}=\frac{A_{t}}{\mu_{2} k_{2} \cos \theta_{2}}
\end{array}\right\}
$$

となり，今のような中間層のない場合と完全に一致する.

次に計算例を示す. Table 1 拉よび 2 は， $V_{2} / V_{1}=4 / 3, \mu_{2} / \mu_{1}=5 / 2$ のとき（このときは $\rho_{2} / \rho_{1}=\mu_{2} V_{1}^{2} / \mu_{1} V_{2}{ }^{2}=45 / 32 \approx 3.6_{5} / 2.6$ となり，ごく普通の地殼の速度，密度分布に相当す る) $, k_{1} H=0,0.4 ， 0.8 ， 1.2$ (これらはそれぞれ $k_{2} H=0,0.3 ， 0.6 ， 0.9$ に対応する）の 場合の反射波，透過波の振幅を計算したもので岁る。即ち Table 1 は媒質 I の側（速度特よ び密度の小さい側）から振幅 1 の波が入射したときの反射波の振幅 $\left|A_{r}\right|$ 抢よび透過波の振幅 $\left|B_{t}\right|$ ， Table 2 は，速度拈よび密度の大きい媒質IIIの側から入射したときの反射波 $\left|B_{r}\right|$ 拈よ び透過波 $\left|A_{t}\right|$ である.

Fig. 2, 3, 4, 5 は，これらを図に示したものであるが，これから見ると， $k_{1} H\left(\right.$ 或いは $\left.k_{2} H\right)$ が大，即ち中間層の厚さに較べて入射波の波長が短いとき，換言すれば，周期の相当短い波は このような中間層の存在により可成影響されることが予想される，殊に反射波は，速度の小さ い側から入射する場合も，大きい側からの場合も， normal incidence のとき，その影響はよ 


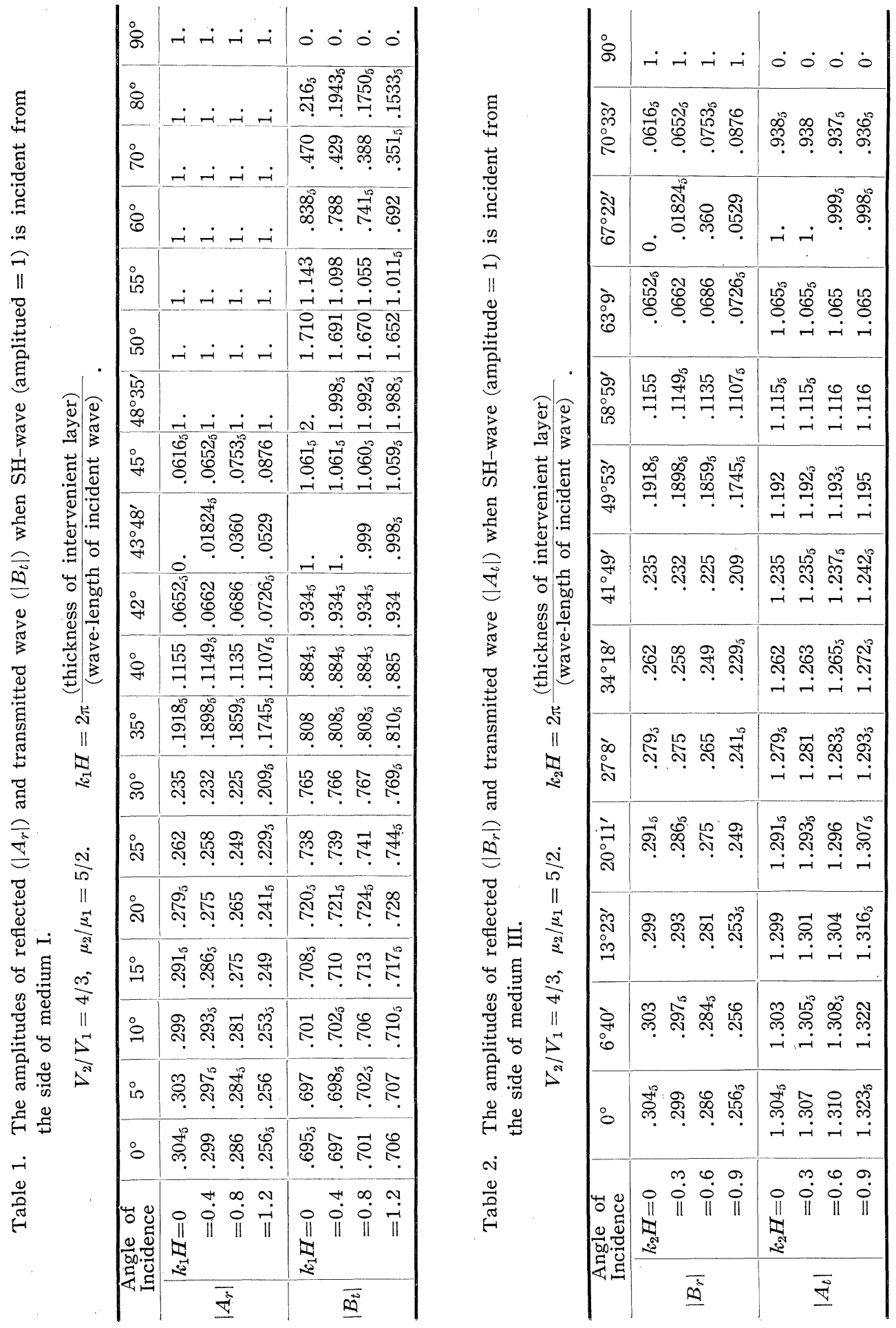




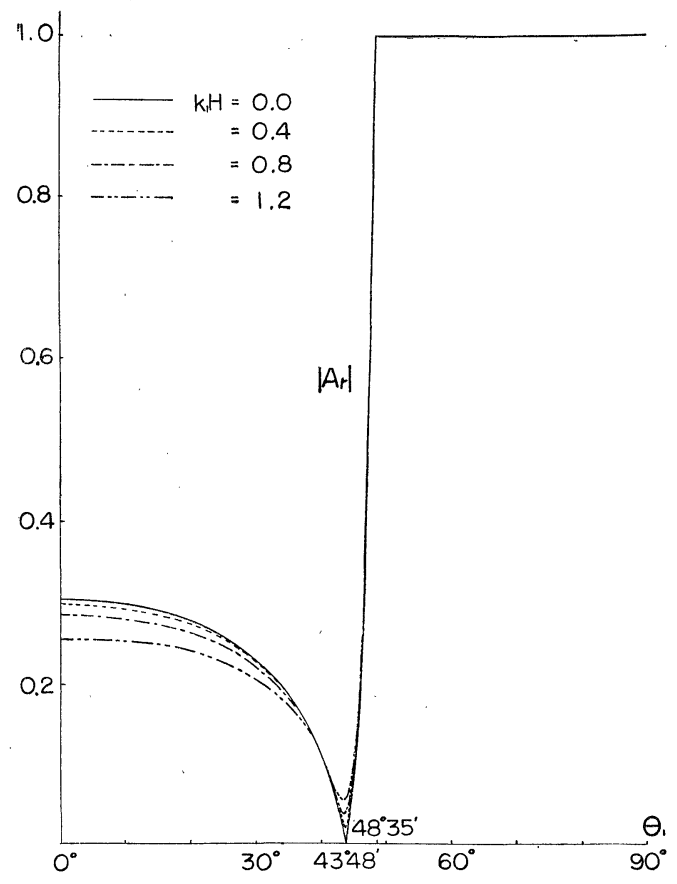

Fig. 2. Amplitude of reflected wave.

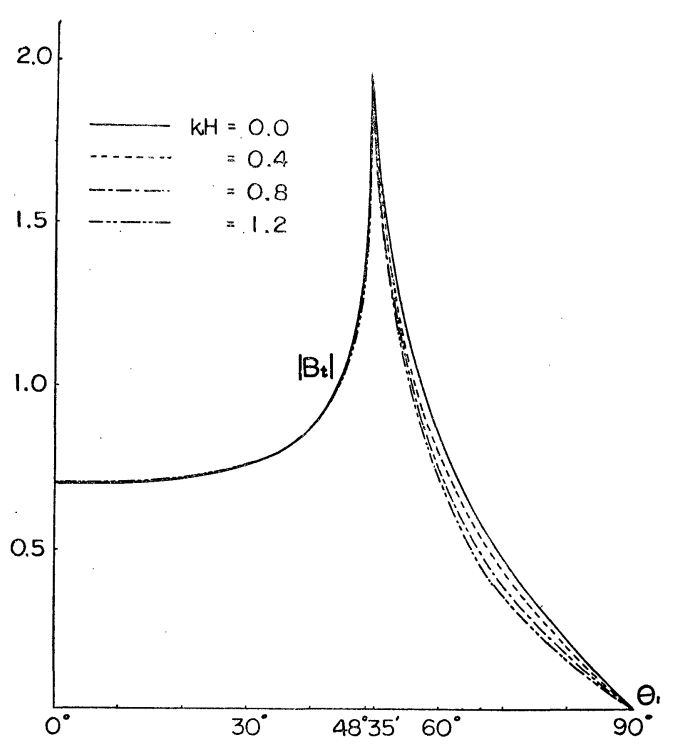

Fig. 3. Amplitude of transmitted wave.

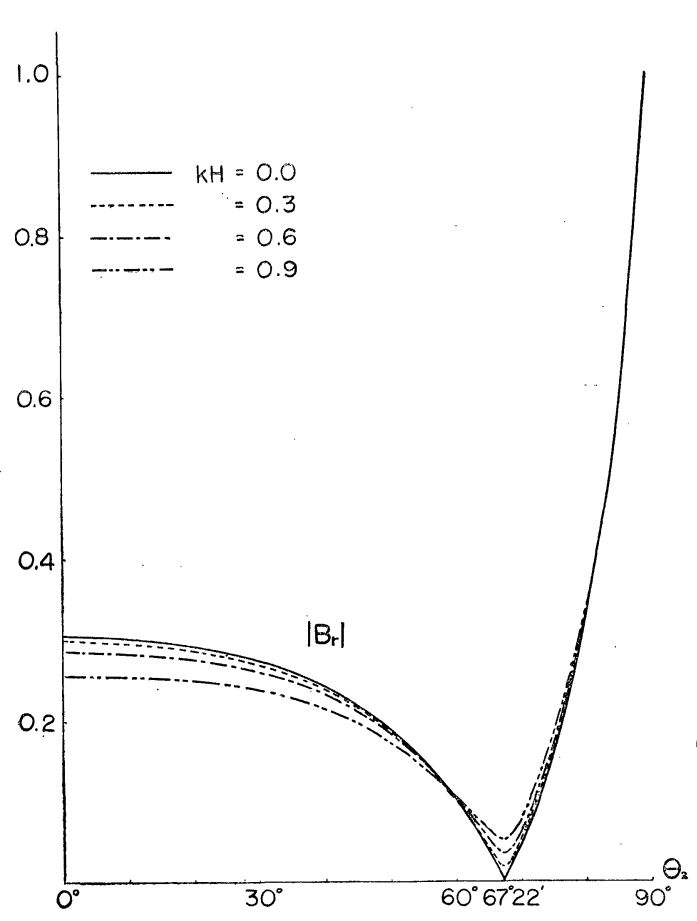

Fig. 4. Amplitude of reflected wave.

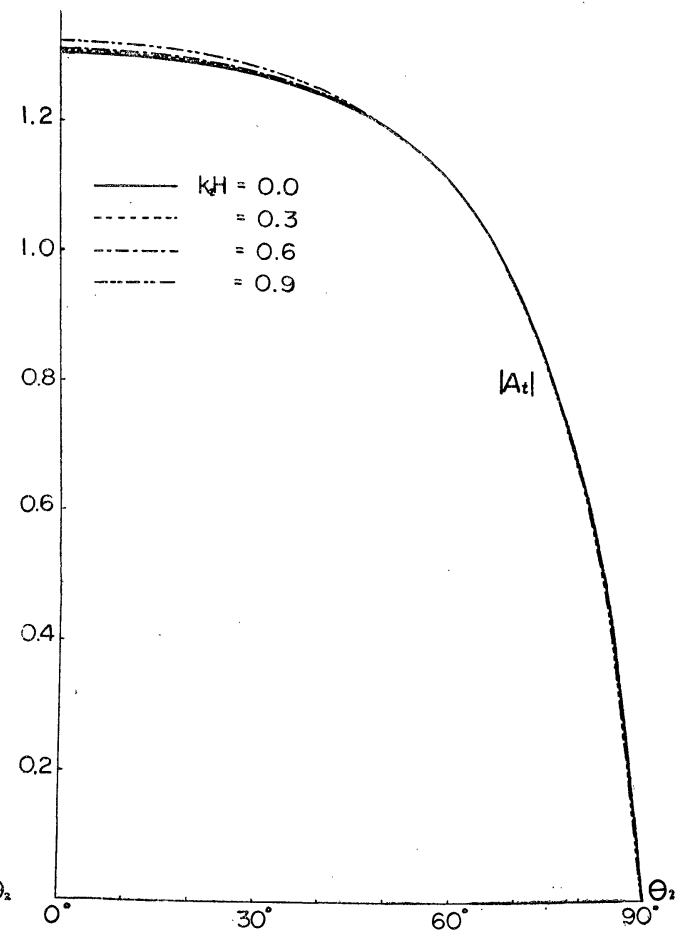

Fig. 5. Amplitude of transmitted wave. 
り大きく，中間層を考学邓場合に較べて振幅が小さくなるようになる，な和，速度の小さい側 から入射する場合，臨界角以上での区射波の振幅は，中間層の有無に影響されず，入射波の振 幅に等しい。

$k_{1} H$ (或いは $\left.k_{2} H\right)$ が上記の值よりずつと大きくなると, 今の法では (26) からも分るよう

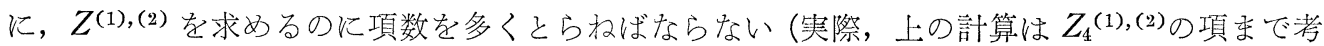
慮しているのであるが， $k_{1} H=1.2$ 位になると，有效数字が 4 桁位である)。攵こで，この場 合は $Z^{(1)}, Z^{(2)}$ として，第 1 種㧊よび第 2 種の Hankel 函数を用い，(22)の解を，

$$
\left.\begin{array}{c}
Z=C Z^{(1)}(\zeta)+D Z^{(2)}(\zeta)=C H_{\nu}^{(1)}(\tau)+D H_{\nu}^{(2)}(\tau) \\
\frac{k_{1}}{b}\left\{1+\left(\begin{array}{c}
a \\
k_{1}
\end{array}\right)^{2}\right\}^{\frac{1}{2}} \geqslant \nu=\frac{p}{q}=\frac{k_{1}}{b}\left\{\sin ^{2} \theta_{1}+\left(\begin{array}{c}
a \\
k_{1}
\end{array}\right)^{2}\right\}^{\frac{1}{2}} \geqslant \frac{a}{b} \\
\frac{k_{1}}{b} \geqslant \tau=\frac{1}{q} e^{-q \zeta}=\frac{k_{1}}{b} e^{-b z} \geqslant \frac{k_{1}}{b} \frac{V_{1}}{V_{2}}
\end{array}\right\}
$$

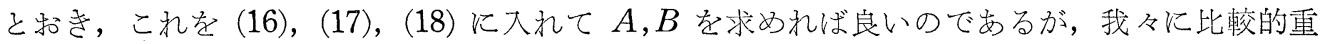
要なのは, normal incidence のときであるので（前述のように，区射波の振幅は，このときょ り強く影響を受けることが予想される)，簡単の為， $\theta_{1}=\theta_{2}=0$ の場合を考光ることにする. このときは $\nu=a / b$ である。

さて, Hankel 函数は, その argument が大きい時, 近似的に,

$$
\left.\begin{array}{l}
H_{\nu}^{(1),(2)}(\tau) \sim \varepsilon e^{ \pm i \Omega}\left[1 \pm\left(\nu^{2}-\frac{1}{4}\right) \frac{i}{2 \tau}+0\left(\frac{1}{\tau^{2}}\right)\right] \\
\frac{d}{d \tau} H_{\nu}^{(1),(2)}(\tau) \sim \pm i \varepsilon e^{ \pm i \Omega}\left[1 \pm\left(\nu^{2}+\frac{3}{4}\right) \frac{i}{2 \tau}+0\left(\frac{1}{\tau^{2}}\right)\right]
\end{array}\right\}
$$

で与えられる。ただし符号は，上が第 1 種，下が第 2 種に対応するものでまた

$$
\varepsilon=\sqrt{2 /(\pi \tau)}, \Omega=\left(\tau-\frac{2 \nu+1}{4} \pi\right),
$$

である. 今 $z=0, H$ に和流る值を，それぞれ suffix $0, H$ を附し， $\tau_{H}=\left(V_{1} / V_{2}\right) \tau_{0}, \varepsilon_{H}=$ $\sqrt{V} V_{2} / V_{1} \varepsilon_{0}$ なることを考慮すると， $k_{1} H$ が $b H$ に較べ相当大きいときは，

$$
\begin{aligned}
& E_{0}^{(1)}=-i \varepsilon_{0} e^{i \Omega_{0}}\left[1+\left(\nu-\frac{1}{2}\right)\left(\nu-\frac{3}{2}\right) \frac{i}{2 \tau_{0}}+0\left(\frac{1}{\tau_{0}^{2}}\right)\right], \\
& E_{0}^{(2)}=i \varepsilon_{0} e^{-i \Omega_{0}}\left[1-\left(\nu-\frac{1}{2}\right)\left(\nu-\frac{3}{2}\right) \frac{i}{2 \tau_{0}}+0\left(\frac{1}{\tau_{0}^{2}}\right)\right] \text {, } \\
& E_{H}^{(1)}=-i \sqrt{\frac{V_{1}}{V_{2}}} \varepsilon_{0} e^{i \Omega_{H}}\left[1+\left(\nu-\frac{1}{2}\right)\left(\nu-\frac{3}{2}\right) \frac{i}{2 \tau_{H}}+0\left(\frac{1}{\tau_{H^{2}}}\right)\right] \text {, } \\
& E_{H}^{(2)}=i \sqrt{\frac{V_{1}}{V_{2}}} \varepsilon_{0} e^{-i \Omega_{H}}\left[1-\left(\nu-\frac{1}{2}\right)\left(\nu-\frac{3}{2}\right) \frac{i}{2 \tau_{H}}+0\left(\frac{1}{\tau_{H^{2}}}\right)\right] \text {. }
\end{aligned}
$$


以上の值から，(18）の各式を $0(1 / \tau)$ まで計算すると，

$$
\begin{aligned}
& X=\sqrt{\frac{V_{2}}{V_{1}} \varepsilon_{0}{ }^{2}}\left[\left\{1-\frac{i}{2 \tau_{0}}\left(\nu-\frac{1}{2}\right)\left(\nu-\frac{3}{2}\right)\left(\frac{V_{2}}{V_{1}}-1\right)\right\} e^{i\left(\Omega_{3}-\Omega_{H}\right)}\right. \\
& \left.-\left\{1+\frac{i}{2 \tau_{0}}\left(\nu-\frac{1}{2}\right)\left(\nu-\frac{3}{2}\right)\left(\frac{V_{2}}{V_{1}}-1\right)\right\} e^{-i\left(\Omega_{0}-\Omega_{H}\right)}\right] \text {, } \\
& Y=\sqrt{\frac{V_{2}}{V_{1}}} \varepsilon_{0}^{2}\left[\left\{1-\frac{i}{2 \tau_{0}}\left(\nu-\frac{1}{2}\right)\left(\nu+\frac{1}{2}\right)\left(\frac{V_{2}}{V_{1}}-1\right)\right\} e^{i\left(\Omega_{0}-\Omega_{H}\right)}\right. \\
& \left.-\left\{1+\frac{i}{2 \tau_{0}}\left(\nu-\frac{1}{2}\right)\left(\nu+\frac{1}{2}\right)\left(\frac{V_{2}}{V_{1}}-1\right)\right\} e^{-i\left(\Omega_{0}-\Omega_{H}\right)}\right] \\
& U=i \sqrt{\frac{V_{2}}{V_{1}}} \varepsilon_{0}^{2}\left[\left\{1-\frac{i}{2 \tau_{0}}\left(\nu-\frac{1}{2}\right)\left(\nu-\frac{3}{2}\right) \frac{V_{2}}{V_{1}}-\left(\nu+\frac{1}{2}\right)\right\} e^{i\left(\Omega_{0}-\Omega_{H}\right)}\right. \\
& \left.+\left\{1+\frac{i}{2 \tau_{0}}\left(\nu-\frac{1}{2}\right)\left(\nu-\frac{3}{2}\right) \frac{V_{2}}{V_{1}}-\left(\nu+\frac{1}{2}\right)\right\} e^{-i\left(\Omega_{0}-\Omega_{H}\right)}\right], \\
& W=-i \sqrt{\frac{V_{2}}{V_{1}}} \varepsilon_{0}^{2}\left[\left\{1-\frac{i}{2 \tau_{0}}\left(\nu-\frac{1}{2}\right)\left(\nu+\frac{1}{2}\right) \frac{V_{2}}{V_{1}}-\left(\nu-\frac{3}{2}\right)\right\} e^{i\left(\Omega_{0}-\Omega_{H}\right)}\right. \\
& +\left\{1+\frac{i}{2 \tau_{0}}\left(\nu-\frac{1}{2}\right) \overline{\left.\left(\nu+\frac{1}{2}\right) \frac{V_{2}}{V_{1}}-\left(\nu-\frac{3}{2}\right)\right\}} e^{-i\left(\Omega_{0}-\Omega_{H}\right)}\right] \text {, } \\
& R=2 i \sqrt{\frac{\mu_{1}}{\mu_{2}}}\left(\frac{V_{2}}{V_{1}}\right) \varepsilon_{0}^{2}, \\
& S=2 i \sqrt{\frac{\mu_{2}}{\mu_{1}}} \varepsilon_{0}{ }^{2} \text {. }
\end{aligned}
$$

また（17）は上式から容易に，

$$
\Delta=-4 \sqrt{\frac{V_{2}}{V_{1}}} \varepsilon_{0}^{2}\left\{1+\frac{i}{2 \tau_{0}}\left(\nu-\frac{1}{2}\right)^{2}\left(\frac{V_{2}}{V_{1}}-1\right)\right\} e^{-i\left(\Omega_{0}-\Omega_{H}\right)} .
$$

かくして， $k_{1} H$ が $b H$ に較べ充分大きいとき， 区射波拈よび透過の振幅は次のように与兄ら れる。

$$
\begin{aligned}
& A_{r} \approx \frac{1}{\Delta^{\prime}} \frac{i(b H)}{2\left(k_{1} H\right)}\left(\frac{a}{b}-\frac{1}{2}\right)\left\{\frac{V_{2}}{V_{1}} e^{2 i\left(\Omega_{0}-\Omega_{H}\right)}-1\right\}, \\
& B_{t} \approx \frac{1}{\Delta^{\prime}} \sqrt{\frac{\mu_{1} V_{2}}{\mu_{2} V_{1}}} e^{i\left(\Omega_{0}-\Omega_{H}\right)}, \\
& B_{r} \approx-\frac{1}{\Delta^{\prime}} \frac{i(b H)}{2\left(k_{1} H\right)}\left(\frac{a}{b}-\frac{1}{2}\right)\left\{e^{2 i\left(\Omega_{0}-\Omega_{H}\right)}-\frac{V_{2}}{V_{1}}\right\}, \\
& A_{\iota} \approx \frac{1}{\Delta^{\prime}} \sqrt{\frac{\mu_{2} V_{1}}{\mu_{1} V_{2}}} e^{i\left(\Omega_{0}-\Omega_{H}\right)} . \\
& \Delta^{\prime}=1+\frac{i(b H)}{2\left(k_{1} H\right)}\left(\frac{a}{b}-\frac{1}{2}\right)^{2}\left(\frac{V_{2}}{V_{1}}-1\right)+0\left\{\left(\frac{b H}{k_{1} H}\right)^{2}\right\} .
\end{aligned}
$$

めるいは, 複素数間の関係 $\left|z_{1}-z_{2}\right| \geqslant\left|z_{1}\right|-\left|z_{2}\right|,\left|z_{1}+z_{2}\right| \leqslant\left|z_{1}\right|+\left|z_{2}\right|$ を利用すると, 


$$
\begin{aligned}
& \left|A_{r}\right| \leqslant \frac{1}{2}\left(\frac{b H}{k_{1} H}\right)\left(\frac{a}{b}-\frac{1}{2}\right)\left(\frac{V_{2}}{V_{1}}+1\right)+0\left\{\left(\frac{b H}{k_{1} H}\right)^{2}\right\}, \\
& \left|B_{t}\right| \geqslant \sqrt{\frac{\mu_{1} V_{2}}{\mu_{2} V_{1}}}\left[1-\frac{1}{2}\left(\frac{b H}{k_{1} H}\right)\left(\frac{a}{b}-\frac{1}{2}\right)^{2}\left(\frac{V_{2}}{V_{1}}-1\right)+0\left\{\left(\frac{b H}{k_{1} H}\right)^{2}\right\}\right], \\
& \left|B_{r}\right| \leqslant \frac{1}{2}\left(\frac{b H}{k_{2} H}\right)\left(\frac{a}{b}-\frac{1}{2}\right)\left(\frac{V_{1}}{V_{2}}+1\right)+0\left\{\left(\frac{b H}{k_{2} H}\right)^{2}\right\}, \\
& \left|A_{t}\right| \geqslant \sqrt{\frac{\mu_{2}}{\mu_{1}} V_{2}}\left[1-\frac{1}{2}\left(\frac{b H}{k_{2} H}\right)\left(\frac{a}{b}-\frac{1}{2}\right)^{2}\left(1-\frac{V_{1}}{V_{2}}\right)+0\left\{\left(\frac{b H}{k_{2} H}\right)^{2}\right\}\right],
\end{aligned}
$$

が得られる。

今, 前に与えたように, $V_{2} / V_{1}=4 / 3, \mu_{2} / \mu_{1}=5 / 2$ とし, また入射波の波長 $L$ と中間層の 厚さ $H$ との比を， $H / L=20 / \pi=6.366 \cdots$ 即ち $k_{1} H$ (或いは $\left.k_{2} H\right)=40$ とすると（このとき $\left.b H / k_{1} H \simeq 0.00719\right)$,

$$
\begin{aligned}
& \left|A_{r}\right| \leqslant 0.00917, \quad\left|B_{t}\right| \geqslant 0.7303(1-0.00143)=0.729_{5}, \\
& \left|B_{r}\right| \leqslant 0.00689_{5}, \quad\left|A_{t}\right| \geqslant 1.3693\left(1-0.00107_{5}\right)=1.368,
\end{aligned}
$$

なる值になる，即ら区射波の方は，波長が短いと，振幅が著しく小さくなることがわかる，上 の例でいうと，波長は中間層の $1 / 6$ 位であるが，このような中間層を考觉ない場合に較べその 振幅は, 速度の小さい方から入射する場合で 0.03 倍位, 大きい方から入射する場合で 0.02 倍 位の大きさになつてしまう。これに区し，透過波の方は，波長の短い方が寧ろ振幅が大きくな るが，非常に短い波でも，いずれも，層を考文場合の高々 1.05 倍位に变るに過ぎないので ある。

\section{§. 結 語}

ここで聂报つたのはSH 波だけであるので，斜めの大射の場合は，P波や SV 波が大射す 当境合についても同じような事がいえるとは勿諭限らない，併し，ここで取报つたような“不

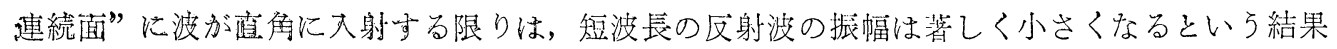
が得られた。

今，色々な周期を含んだ波が，このような不連続面にほぼ直角に入射するときを考光ると， ここで取扱つたような中間漸移層は，区射波に対しては一種の low pass filter のような作用 をし，直接波に較べて反射波の凰期が長くなるという現象が見られるわけである。斜めに大射 する場合は，必ずしもこのようなことは見られない。

最後に, 終始御指導, 御鞭撻頂いた松沢教授执よび松沢研究室の皆様に厚く感謝する. 
参考文 献

1) C. K. Knott; Phil. Mag., [V], 45 (1899), 64 97.

2) K. Sezawa and K. Kanai; Bull. Earthq. Res. Inst., 13 (1935), 750 756.

3) A. Wolf; Geophysics, 2 (1937), 357 363. 